\title{
GOLD THERAPY AND NEUTRAL 17-KETOSTEROID EXCRETION IN RHEUMATOID ARTHRITIS
}

\author{
BY
}

\author{
JAMES BRUCE and ROBERT MACKAY \\ Royal Northern Infirmary, Inverness
}

(RECEIVED FOR PUBLICATION JULY 14, 1952)

\begin{abstract}
Although the striking benefits of the administration of cortisone and ACTH in rheumatoid arthritis have been widely publicized, the exhibition of gold salts still retains an important place in the therapy of some types of case. The manner in which gold salts act is not known, but has been ascribed to stimulation of the reticulo-endothelial system, some alteration in the immunological or enzymatic mechanisms, or to bacteriostasis. Since a relationship appears to exist between rheumatoid arthritis and steroid metabolism, it was felt that it would be of interest to investigate what changes, if any, take place in the urinary excretion of neutral 17-ketosteroids in cases selected for gold therapy.
\end{abstract}

The cases selected by the clinician for gold therapy were those in which improvement could reasonably be expected, the degree of activity of the disease process being assessed by clinical examination and the erythrocyte sedimentation rate. Sixteen cases, six male and ten female, were investigated, four of the females being post-menopausal.

In hospital out-patients, 24-hr collections of urine were examined for neutral 17-ketosteroid excretion twice weekly; when cases were under observation in hospital, daily determinations were made at first, but these were reduced to twice-weekly also when it became apparent that there was no immediate change in the daily pattern of excretion. Immediately before treatment in each case the 24-hr excretion was determined on each of three successive days to establish a base line. With the exception of one male (Case I) and one female (Case II) there was improvement, ranging in degree from slight to well-marked, in all patients investigated.

\section{Methods}

Neutral 17-ketosteroids were determined by the Callow-Zimmermann method following the standard technique recommended by the Medical Research iv Council Committee on Clinical Endocrinology (1951).

\section{Results}

The 24-hr excretion of neutral 17-ketosteroids $\mathscr{\oplus}$ before, during, and after therapy (twice-weekly $\frac{\mathbb{O}}{\mathbb{O}}$ estimations) is given in $\mathrm{mg}$. in the Table opposite.

\section{Discussion}

These results indicate that chrysotherapy does no $8 \overrightarrow{0}$ produce an appreciable change in 17-ketosteroid. excretion level. Davison, Koets, and Kuzell (194 and Wolfson and others (1949) found the excretion of 17-ketosteroids in rheumatoid arthritis to be within the normal range, and our base-line levels agree with their results. It may be noted, however, that in some of our cases the figures approach the lower limit of normal-findings which have already been observed by Hench and others (1949). The results of these other workers, as Marrian (1951) observed, do not lend support to the idea that in rheumatoid arthritis there is gross disorder in the secretion or metabolism of the adrenocortical hormones. Were this condition primarily due to dysfunction of the adrenal cortex, one might expect effective therapy to be reflected in some alteration in the output of 17-ketosteroids-if the excretion of these can be taken as a measure of adrenocortical activity-and the relative stability of excretion levels in our series seems further to strengthen the view expressed by Marrian. How gold acts in rheumatoid arthritis remains obscure, but our results suggest that in cases deriving benefit from its administration the improvement cannot be attributed to any action on the adrenal cortex.

\section{Summary}

The excretion of neutral 17-ketosteroids was 
TABLE

24-HR NEUTRAL 17-KETOSTEROID EXCRETION (BI-WEEKLY) IN SIX MALE AND TEN FEMALE PATIENTS

\begin{tabular}{|c|c|c|c|c|c|c|c|c|c|c|c|c|c|c|c|c|}
\hline \multirow{2}{*}{$\begin{array}{l}\text { Weeks } \\
\text { of } \\
\text { Therapy }\end{array}$} & \multicolumn{10}{|c|}{ Female Cases } & \multicolumn{6}{|c|}{ Male Cases } \\
\hline & I & II & III & IV & $\mathbf{V}$ & VI & VII & VIII & IX & $\mathbf{x}$ & I & II & III & IV & $\mathbf{V}$ & VI \\
\hline 0 & $3 \cdot 0$ & $6 \cdot 1$ & $3 \cdot 3$ & $3 \cdot 2$ & $9 \cdot 0$ & 6.9 & $11 \cdot 8$ & 8.4 & $2 \cdot 8$ & $7 \cdot 6$ & $8 \cdot 1$ & 6.9 & $10 \cdot 2$ & 6.4 & $4 \cdot 2$ & $7 \cdot 3$ \\
\hline \multirow[t]{2}{*}{1} & $3 \cdot 0$ & $4 \cdot 6$ & $5 \cdot 1$ & $4 \cdot 1$ & $7 \cdot 8$ & $8 \cdot 6$ & $12 \cdot 3$ & $8 \cdot 7$ & $3 \cdot 3$ & $7 \cdot 2$ & $7 \cdot 9$ & $6 \cdot 6$ & $11 \cdot 0$ & $7 \cdot 7$ & 2.9 & 7.9 \\
\hline & $4 \cdot 4$ & 5.9 & $3 \cdot 1$ & $3 \cdot 8$ & $9 \cdot 4$ & $9 \cdot 2$ & $10 \cdot 4$ & $7 \cdot 4$ & $3 \cdot 1$ & $7 \cdot 8$ & $7 \cdot 3$ & $7 \cdot 7$ & $11 \cdot 2$ & $7 \cdot 9$ & $2 \cdot 6$ & $7 \cdot 1$ \\
\hline \multirow{2}{*}{2} & $3 \cdot 8$ & $6 \cdot 3$ & $3 \cdot 0$ & $4 \cdot 5$ & $8 \cdot 5$ & $8 \cdot 4$ & $10 \cdot 8$ & $7 \cdot 9$ & & $8 \cdot 4$ & $8 \cdot 4$ & $7 \cdot 4$ & $10 \cdot 9$ & $6 \cdot 0$ & $3 \cdot 1$ & $6 \cdot 7$ \\
\hline & 4.6 & 6.7 & $2 \cdot 4$ & $3 \cdot 7$ & $11 \cdot 0$ & $7 \cdot 8$ & $11 \cdot 1$ & $8 \cdot 2$ & 2.9 & $7 \cdot 6$ & 8.8 & 8.6 & $9 \cdot 3$ & $7 \cdot 2$ & $3 \cdot 8$ & $7 \cdot 8$ \\
\hline \multirow{2}{*}{3} & $3 \cdot 2$ & $6 \cdot 5$ & $5 \cdot 0$ & & $9 \cdot 7$ & $7 \cdot 5$ & & $8 \cdot 7$ & $3 \cdot 5$ & $7 \cdot 4$ & $7 \cdot 9$ & $8 \cdot 0$ & $11 \cdot 2$ & $6 \cdot 1$ & $3 \cdot 1$ & $7 \cdot 1$ \\
\hline & $4 \cdot 3$ & 6.9 & $3 \cdot 4$ & & $11 \cdot 0$ & $7 \cdot 0$ & $12 \cdot 5$ & $9 \cdot 3$ & $3 \cdot 4$ & $7 \cdot 0$ & $9 \cdot 1$ & $8 \cdot 2$ & $10 \cdot 7$ & $8 \cdot 2$ & $4 \cdot 6$ & $6 \cdot 4$ \\
\hline \multirow{2}{*}{4} & & $5 \cdot 2$ & 3.9 & & 8.4 & $8 \cdot 3$ & $11 \cdot 9$ & & $2 \cdot 7$ & & $8 \cdot 5$ & $7 \cdot 5$ & $9 \cdot 6$ & $5 \cdot 2$ & 3.8 & $5 \cdot 7$ \\
\hline & 4.8 & $7 \cdot 9$ & 3.6 & $2 \cdot 5$ & $8 \cdot 3$ & $8 \cdot 7$ & & $9 \cdot 1$ & $2 \cdot 6$ & 6.9 & $7 \cdot 2$ & $7 \cdot 2$ & $10 \cdot 6$ & $7 \cdot 3$ & 5.5 & 6.9 \\
\hline \multirow{2}{*}{5} & $3 \cdot 8$ & $6 \cdot 1$ & $4 \cdot 2$ & $5 \cdot 1$ & $9 \cdot 7$ & $7 \cdot 4$ & $11 \cdot 4$ & $8 \cdot 3$ & $3 \cdot 8$ & $8 \cdot 2$ & $7 \cdot 7$ & $6 \cdot 3$ & 8.8 & $5 \cdot 8$ & $5 \cdot 2$ & 8.0 \\
\hline & 2.9 & 6.6 & 3.7 & 4.8 & $10 \cdot 8$ & $8 \cdot 4$ & $12 \cdot 1$ & & 3.9 & $8 \cdot 8$ & 8.8 & 7.6 & 9.0 & 6.4 & 5.8 & $5 \cdot 5$ \\
\hline \multirow{2}{*}{6} & 4.0 & 5.4 & $4 \cdot 0$ & $4 \cdot 1$ & 8.6 & 8.0 & $12 \cdot 2$ & $8 \cdot 1$ & 2.9 & 6.4 & 6.9 & $7 \cdot 7$ & $11 \cdot 1$ & $5 \cdot 7$ & 3.6 & $7 \cdot 9$ \\
\hline & $2 \cdot 4$ & 6.8 & $3 \cdot 3$ & $4 \cdot 2$ & $10 \cdot 5$ & & $11 \cdot 4$ & 9.4 & $4 \cdot 1$ & 6.8 & $7 \cdot 3$ & $8 \cdot 3$ & 8.6 & $7 \cdot 5$ & 5.6 & 5.8 \\
\hline \multirow{2}{*}{7} & 3.0 & $7 \cdot 1$ & & 3.7 & $7 \cdot 7$ & $7 \cdot 3$ & & $9 \cdot 0$ & 4.0 & $7 \cdot 2$ & $8 \cdot 2$ & 6.9 & $10 \cdot 5$ & 7.6 & 3.4 & 6.7 \\
\hline & $3 \cdot 2$ & 6.9 & 2.9 & $4 \cdot 7$ & 7.9 & $7 \cdot 9$ & $11 \cdot 2$ & $7 \cdot 2$ & $2 \cdot 3$ & $7 \cdot 8$ & 8.9 & $7 \cdot 2$ & 9.4 & $7 \cdot 9$ & $4 \cdot 8$ & 5.6 \\
\hline \multirow{2}{*}{8} & $3 \cdot 8$ & $6 \cdot 3$ & 3.6 & 3.0 & 8.6 & $7 \cdot 2$ & $10 \cdot 9$ & $7 \cdot 3$ & $2 \cdot 8$ & $8 \cdot 3$ & 8.0 & $5 \cdot 8$ & $10 \cdot 9$ & 5.0 & $5 \cdot 1$ & $7 \cdot 4$ \\
\hline & & $7 \cdot 1$ & 3.9 & $3 \cdot 8$ & 8.7 & 6.9 & $12 \cdot 0$ & 8.7 & $2 \cdot 3$ & 6.4 & $7 \cdot 6$ & $5 \cdot 8$ & 8.9 & 6.5 & 4.9 & $8 \cdot 2$ \\
\hline \multirow{2}{*}{9} & & 6.6 & $4 \cdot 4$ & 1.9 & 8.0 & $8 \cdot 1$ & $10 \cdot 9$ & & $2 \cdot 5$ & 8.0 & $9 \cdot 8$ & $7 \cdot 7$ & $9 \cdot 6$ & 8.0 & 4.0 & 6.1 \\
\hline & 2.6 & 6.3 & 2.9 & 3.6 & $10 \cdot 2$ & 6.9 & $11 \cdot 2$ & $8 \cdot 3$ & 2.5 & & $8 \cdot 2$ & 6.4 & $11 \cdot 2$ & 6.9 & 6.0 & $5 \cdot 8$ \\
\hline \multirow{2}{*}{10} & $3 \cdot 7$ & 5.9 & 3.6 & 4.9 & & $7 \cdot 4$ & $12 \cdot 1$ & $9 \cdot 3$ & $3 \cdot 3$ & $8 \cdot 2$ & 8.9 & 7.9 & $11 \cdot 1$ & $7 \cdot 7$ & $4 \cdot 7$ & $8 \cdot 1$ \\
\hline & $2 \cdot 8$ & $5 \cdot 8$ & 3.6 & & $9 \cdot 8$ & $7 \cdot 2$ & $11 \cdot 3$ & 9.0 & $3 \cdot 0$ & $7 \cdot 0$ & $7 \cdot 4$ & $7 \cdot 2$ & $9 \cdot 2$ & 6.0 & $5 \cdot 2$ & $7 \cdot 5$ \\
\hline \multirow{2}{*}{11} & 4.4 & $6 \cdot 2$ & & & & $6 \cdot 7$ & $11 \cdot 7$ & 7.9 & $2 \cdot 9$ & $7 \cdot 9$ & $8 \cdot 8$ & $6 \cdot 8$ & $\begin{array}{r}10 \cdot 3 \\
9 \cdot 6 \\
\end{array}$ & $5 \cdot 8$ & $2 \cdot 8$ & 6.8 \\
\hline & 3.9 & 6.9 & $4 \cdot 8$ & & $9 \cdot 3$ & 6.4 & 10.9 & $7 \cdot 3$ & & $8 \cdot 1$ & 6.7 & $7 \cdot 5$ & 8.7 & $6 \cdot 3$ & 3.6 & 7.9 \\
\hline \multirow{2}{*}{12} & 5.0 & 6.0 & $3 \cdot 2$ & $3 \cdot 8$ & 8.9 & $8 \cdot 0$ & $12 \cdot 1$ & 9.0 & $3 \cdot 3$ & $8 \cdot 1$ & 8.4 & 7.9 & $8 \cdot 6$ & 5.9 & $4 \cdot 2$ & 5.9 \\
\hline & $3 \cdot 7$ & $5 \cdot 3$ & 5.0 & & $10 \cdot 1$ & 6.9 & $12 \cdot 4$ & & 3.6 & & $7 \cdot 9$ & $8 \cdot 1$ & $9 \cdot 8$ & $5 \cdot 8$ & 5.9 & $7 \cdot 0$ \\
\hline \multirow{2}{*}{13} & $4 \cdot 6$ & $5 \cdot 8$ & 4.7 & & 8.9 & 6.4 & $10 \cdot 8$ & & $2 \cdot 7$ & $7 \cdot 3$ & & & & & & \\
\hline & & 6.4 & 4.7 & & 7.9 & $7 \cdot 0$ & $11 \cdot 7$ & & & 6.9 & & & & & & \\
\hline \multirow{2}{*}{14} & & 5.9 & $3 \cdot 3$ & & $8 \cdot 2$ & & $11 \cdot 0$ & & & 8.0 & & & & & & \\
\hline & $2 \cdot 8$ & 6.6 & 3.7 & & $7 \cdot 8$ & $7 \cdot 2$ & $12 \cdot 1$ & & & $6 \cdot 8$ & & & & & & \\
\hline \multicolumn{17}{|c|}{ Weeks after Therapy } \\
\hline \multirow{2}{*}{2} & $3 \cdot 7$ & 5.9 & 3.7 & & $10 \cdot 2$ & 6.7 & & & $2 \cdot 0$ & $8 \cdot 3$ & 8.9 & $8 \cdot 2$ & $9 \cdot 2$ & $7 \cdot 6$ & 6.0 & 6.7 \\
\hline & $4 \cdot 3$ & $6 \cdot 3$ & $3 \cdot 2$ & $2 \cdot 1$ & $9 \cdot 7$ & $7 \cdot 5$ & $12 \cdot 9$ & & & $7 \cdot 9$ & $7 \cdot 8$ & $7 \cdot 6$ & 9.9 & $6 \cdot 1$ & $4 \cdot 5$ & $8 \cdot 4$ \\
\hline \multirow{2}{*}{4} & & 6.0 & $3 \cdot 2$ & 4.4 & $10 \cdot 6$ & $8 \cdot 2$ & $10 \cdot 6$ & $7 \cdot 8$ & $3 \cdot 2$ & $7 \cdot 4$ & 8.0 & $5 \cdot 8$ & 8.9 & 6.9 & $5 \cdot 3$ & 5.9 \\
\hline & 4.9 & & $4 \cdot 1$ & & $9 \cdot 1$ & 7.9 & $11 \cdot 4$ & & & 8.6 & $6 \cdot 8$ & 8.0 & $10 \cdot 8$ & $5 \cdot 5$ & $3 \cdot 8$ & 8.6 \\
\hline \multirow{2}{*}{6} & $3 \cdot 8$ & $5 \cdot 8$ & $4 \cdot 0$ & & $7 \cdot 8$ & $6 \cdot 8$ & $12 \cdot 5$ & & & & $7 \cdot 6$ & $8 \cdot 2$ & $9 \cdot 3$ & $5 \cdot 8$ & $5 \cdot 6$ & $6 \cdot 4$ \\
\hline & & $6 \cdot 3$ & $3 \cdot 3$ & & 8.4 & $6 \cdot 1$ & & & $4 \cdot 0$ & $8 \cdot 9$ & $7 \cdot 2$ & $7 \cdot 7$ & $11 \cdot 4$ & $5 \cdot 3$ & $5 \cdot 2$ & $8 \cdot 3$ \\
\hline
\end{tabular}

observed in six male and ten female patients with rheumatoid arthritis during and after gold treatment.

No marked changes are reported. The signi- ficance of these results is discussed, especially as regards their bearing on adrenocortical activity, which does not seem to have been much affected, 
although the patients' arthritis was improved by the gold treatment.

The majority of these cases were under the care of Dr. D. C. Wilson, to whom we are grateful for allowing us to investigate them.

\section{REFERENCES}

Davison, R. A., Koets, P., and Kuzell, W. C. (1947). J. clin.

Endocrinol., 7, 201. Proc. Mayo Clin., 24, 181.

Marrian, G. F. (1951). Practitioner, 166, 43.

Medical Research Council Committee on Clinical Endocrinology (1951). Lancet, 2, 585.

Wolfson, W. Q., Guterman, H. S., Levine, R., Cohn, C., Hunt, H. D., and Rosenberg, E. F. (1949). J. clin. Endocrinol., 9, 497.

Chrysothérapie et l'excrétion de 17-cetostéroìdes neutres dans l'arthrite rhumatismale

RÉSUMÉ

L'excrétion de 17-cetostéroïdes neutres fut étudiée chez six hommes et dix femmes atteints d'arthrite rhumatismale pendant et après le traitement par des
sels d'or.

On ne trouva pas d'altérations importantes. Les [ auteurs discutent la portée de ces résultats, surtout par rapport à l'activité cortico-surrénale, qui ne semble pas $\Rightarrow$ avoir été très affectée, malgré l'amélioration de l'arthrite par la chrysothérapie.

La auroterapia y la excreción de 17-cetosteroides neutros en la artritis reumatoide

\section{Sumario}

La excreción de 17-cetosteroides neutros fué estudiada $\vec{\circ}$ en seis hombres y diez mujeres con artritis reumatoide, durante y después del tratamiento con sales de oro.

No se hallaron alteraciones importantes. Se discute $\stackrel{\sigma}{\circ}$ lo significado de estos resultados, especialmente respecto $\cong$ a la actividad córtico-suprarrenal, que no parece haber 0 sido muy afectada, a pesar del efecto favorable de la $\overrightarrow{-}$ auroterapia sobre la artritis. 